\title{
Detection of Vertebral Body Fractures Based on Cortical Shell Unwrapping
}

\author{
Jianhua Yao ${ }^{1}$, Joseph E. Burns ${ }^{2}$, Hector Munoz ${ }^{1}$, and Ronald M. Summers ${ }^{1}$ \\ ${ }^{1}$ Radiology and Imaging Sciences Department, Clinical Center, The National Institutes \\ of Health, Bethesda, MD 20892 \\ ${ }^{2}$ Department of Radiological Sciences, University of California, Irvine School of Medicine
}

\begin{abstract}
Assessment of trauma patients with multiple injuries can be one of the most clinically challenging situations dealt with by the radiologist. We propose a fully automated method to detect acute vertebral body fractures on trauma CT studies. The spine is first segmented and partitioned into vertebrae. Then the cortical shell of the vertebral body is extracted using deformable dual-surface models. The extracted cortical shell is unwrapped onto a 2D map effectively converting a complex 3D fracture detection problem into a pattern recognition problem of fracture lines on a $2 \mathrm{D}$ plane. Twenty-eight features are computed for each fracture line and sent to a committee of support vector machines for classification. The system was tested on 18 trauma CT datasets and achieved $95.3 \%$ sensitivity and 1.7 false positives per case by leave-one-out cross validation.
\end{abstract}

\section{Introduction}

Assessment of trauma patients with multiple injuries, particularly in the setting of multiple trauma patients presenting to the hospital concurrently, can be one of the most clinically challenging situations dealt with by the radiologist. Traumatic injury of the spine is a subset of the spectrum of blunt trauma pathology, and is common and potentially devastating. Previous reports estimate the number of vertebral fractures each year in the United States at more than 140,000, with 19\%-50\% of fractures of the thoracolumbar spine associated with neurological injury [1]. Rapid and accurate assessment is essential for determination of an acceptable management algorithm, and delay in detection and management of spinal injuries can result in prolonged pain and suffering, or biomechanical disability.

Limited forays have been performed in prior work investigating computer-aided assessment of fractures, mainly in the realm of the detection of fractures on plain film radiographs for limited clinical circumstances [2]. Design of algorithms has been performed for simple assessment of anterior height loss of thoracolumbar vertebrae, and has recently reached the stage of clinical application limited to plain film radiograph lateral views of the spine [3]. There are also prior works assessing for fractures based on detected global geometric deformities of the vertebral bodies (compression deformities), rather than direct detection of fracture lines in the vertebrae [4] [5]. Analysis of the complex structure of the spine on cross sectional CT images for direct visualization of fractures is a novel topic of clinical importance, and is the goal and focus of this investigation. To the best of our knowledge, our 
investigation is the first to target computer-aided detection of fracture-associated bone discontinuities in vertebral bodies using CT images.
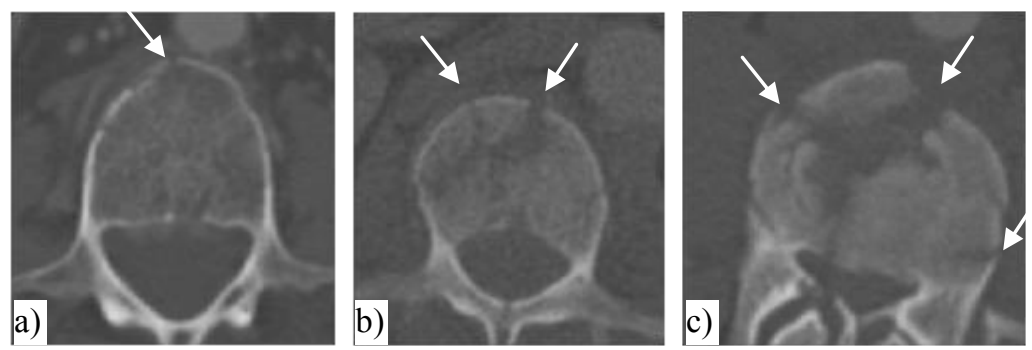

Fig. 1. Examples of vertebral body fractures (arrows) on axial CT images in three different patients

Figure 1 shows examples of vertebral body fractures in axial CT images. Fractures occur in myriad patterns, and vertebrae exhibit complex geometry, posing a complex mathematical problem. Our idea is to directly detect the osseous fracture line involvement of the anterior, posterior, and lateral cortices of the vertebral body. Thus, we propose a novel method to convert the complex $3 \mathrm{D}$ detection problem into a $2 \mathrm{D}$ pattern recognition problem by unwrapping the cortical shell of the vertebral body.

\section{Methods}

Our method is summarized as follows. Given a spine CT data set, the spinal column is first extracted and partitioned into individual vertebrae. The cortical shell of vertebral body is then segmented using deformable dual-surface models. After that, the cortical shell is unwrapped onto a 2D plane. Pattern recognition techniques are then applied to detect fracture lines on the unwrapped cortical shell. These detections are then reprojected back to $3 \mathrm{D}$ space and quantitative features are computed. Finally, the detections are passed to a committee of support vector machines for classification.

\subsection{Spinal Column Segmentation and Partitioning}

First, thresholding and connected component analysis are conducted to obtain the initial spine segmentation. The spinal canal is then extracted using a watershed algorithm and a directed acyclic graph search. Next, curved planar reformation is computed along the centerline of the spinal canal to partition the spinal column into individual vertebrae. Details of the automated spinal column extraction and partitioning can be found in [6].

\subsection{Cortical Shell Segmentation}

In a fractured vertebral body, the cortical shell is often damaged with cracks or broken into disconnected components, posing challenges for its segmentation. Concentric ring approach can't segment cortical shell correctly. We propose a deformable dual-surface model to extract both the exterior and interior (periosteal and endosteal) surfaces of the cortical shell. 

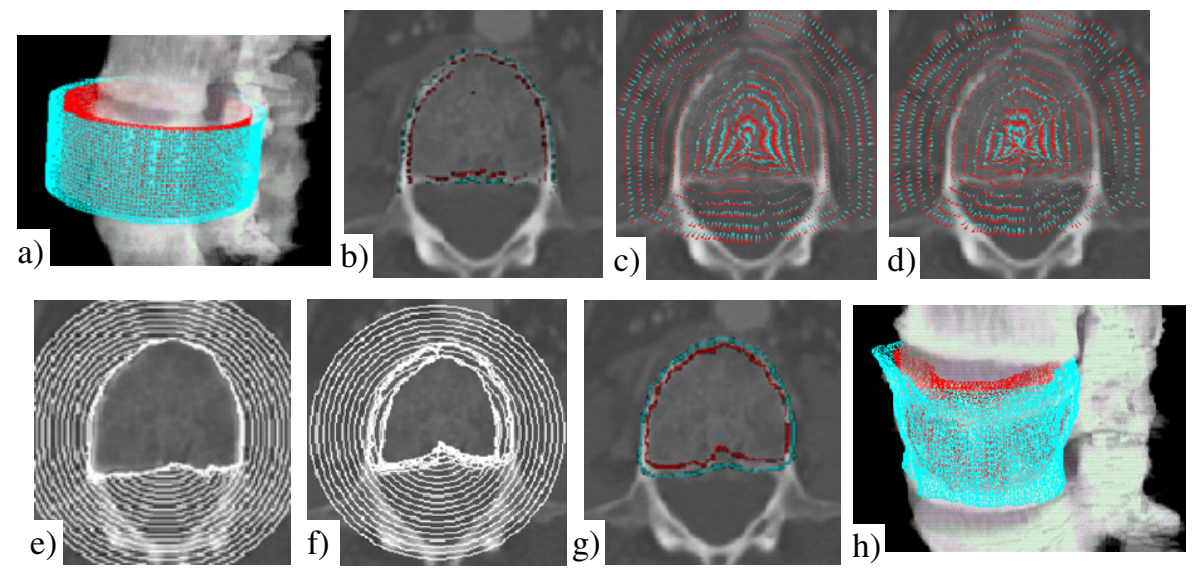

Fig. 2. Cortical shell segmentation. The original image is in Figure 1a. a) Initial models; b) Potential boundary map $\left(R_{E}, R_{I}\right)$, cyan: $R_{E}$, red: $R_{I}$ c) Potential force for exterior surface $P\left(S_{E}\right)$; d) Potential force for interior surface $P\left(S_{I}\right)$. Maps are down-sampled for clarity. Force direction points from red to cyan; e) Evolution of exterior surface; f) Evolution of interior surface; g) Results of dual-surface segmentation; and h) 3D visualization.

After the spine is extracted, a local cylindrical coordinate system is established for each vertebral body. An initial dual surface is placed in the center of the vertebral body. The height is set as the distance between the superior and inferior end plates and the radius is estimated as twice of the average width of the vertebral body (Figure $2 a)$. The surface is constructed as a triangular mesh, where the vertices are evenly spaced. The surface can be represented as $r=S(z, \varphi)$ in the cylindrical coordinate system, where $z$ is the height along the axis, $\varphi$ is the azimuth angle, and $r$ is the radial distance. $r$ is uniform at every point on the initial exterior $\left(S_{E}\right)$ and interior $\left(S_{I}\right)$ surfaces. The resolution of the surface mesh is set to be the same as the CT image.

The deformable dual-surfaces [7] are driven by internal forces, image potential forces, and constraints between the exterior and interior surfaces. The energy functional for the dual-surface is written as,

$$
\begin{aligned}
& \mathrm{E}\left(S_{E}\right)=w_{i} I\left(S_{E}\right)+w_{p} P\left(S_{E}\right)+w_{c} C\left(S_{E}, S_{I}\right) \\
& \mathrm{E}\left(S_{I}\right)=w_{i} I\left(S_{I}\right)+w_{p} P\left(S_{I}\right)+w_{c} C\left(S_{E}, S_{I}\right)
\end{aligned}
$$

where $S_{E}$ and $S_{I}$ are exterior and interior surfaces, $I(S)$ is the internal force, $P(S)$ is the image potential force, $C\left(S_{E}, S_{I}\right)$ is the constraint between the two surfaces, and $w_{i}, w_{p}$ and $w_{c}$ are weights for the three forces. The internal forces keep the surface smooth and continuous, which can be written as,

$$
I(S(z, \varphi))=\int\left(\alpha\left|\frac{\partial S(z, \varphi)}{\partial z}+\frac{\partial S(z, \varphi)}{\partial \varphi}\right|+\beta\left|\frac{\partial^{2} S(z, \varphi)}{\partial z^{2}}+\frac{\partial^{2} S(z, \varphi)}{\partial \varphi^{2}}+\frac{\partial^{2} S(z, \varphi)}{\partial z \partial \varphi}\right|\right) d z d \varphi
$$

The first order derivative discourages the stretching and the second order derivative discourages the bending of the surfaces. $\alpha$ and $\beta$ are weights and set to 1 . 
A directional gradient in the cylindrical coordinate system is applied to compute the potential image. For a point $(z, \varphi, r)$ on the image, the directional gradient is

$$
\nabla \vec{G}(z, \varphi, r)=\frac{\partial G(z, \varphi, r)}{\partial r}
$$

where $G$ is the grayscale image. For every direction defined by $(z, \varphi)$ (at level $z$, angle $\varphi$ ), we search for the maximum of a pair of positive and negative directional gradients to be used as the potential boundary for exterior and interior surfaces, i.e.,

$$
\left(\begin{array}{c}
R_{E}(z, \varphi) \\
R_{I}(z, \varphi)
\end{array}\right)=\underset{\substack{r_{E}, r_{I} \\
s t, r_{I}<r_{E}, \varepsilon_{1}<r_{E}-r_{I}<\varepsilon_{2}}}{\arg \max }\left(\left|\nabla \vec{G}^{+}\left(z, \varphi, r_{E}\right)\right|+\left|\nabla \vec{G}^{-}\left(z, \varphi, r_{I}\right)\right|\right)
$$

Here $\nabla \vec{G}^{+}$and $\nabla \vec{G}^{-}$represent positive and negative directional gradient respectively, and $\varepsilon_{1}$ and $\varepsilon_{2}$ are the minimum and maximum cortical shell thickness. Due to the image noise and other anatomical structures near the vertebral body, $\left(R_{E}\right.$, $R_{I}$ ) may become stuck at false edges. In order to eliminate outliers, we fit a Bezier function for $R_{E}$ over the domain of $(z, \varphi)$. Those $\left(R_{E}, R_{I}\right)$ pairs that are far away from the Bezier function are excluded. Figure $2 \mathrm{~b}$ shows an example of $\left(R_{E}, R_{I}\right)$ map superimposed on an image slice. The distance to the $\left(R_{E}, R_{I}\right)$ map is then used to derive the potential force for the dual surfaces, which can be formulated as,

$$
\begin{aligned}
& P\left(S_{E}(z, \varphi)\right)=\left\|S_{E}(z, \varphi)-R_{E}(z, \varphi)\right\| \\
& P\left(S_{I}(z, \varphi)\right)=\left\|S_{I}(z, \varphi)-R_{I}(z, \varphi)\right\|
\end{aligned}
$$

where \|\| is the Euclidean distance. Figures $2 \mathrm{c}$ and $2 \mathrm{~d}$ show the potential forces $P\left(S_{E}\right)$ and $P\left(S_{I}\right)$.

The constraint between the dual surfaces is the thickness of the cortical shell. We assume that the thickness should be continuous over the cortical shell, and use the following function for the thickness regulation,

$$
C\left(S_{E}, S_{I}\right)=\int\left(\left|\frac{\partial\left(S_{E}(z, \varphi)-S_{I}(z, \varphi)\right)}{\partial z}+\frac{\partial\left(S_{E}(z, \varphi)-S_{I}(z, \varphi)\right)}{\partial \varphi}\right|\right) d z d \varphi
$$

The weights for different forces $\left(w_{i}, w_{p}\right.$ and $\left.w_{c}\right)$ in equation 1 are kept constant throughout the evolution. Since the potential force becomes smaller when closer to the boundaries (see Fig. 2c and 2d), the internal force and thickness constraint will play a bigger role upon convergence. Figures $2 \mathrm{e}$ and $2 \mathrm{f}$ show the evolution of the exterior and interior surfaces. Figures $2 \mathrm{~g}$ and $2 \mathrm{~h}$ show the final segmentation results on one 2D slice and in 3D space.

\subsection{Cortical Shell Unwrapping}

The unwrapping of the cortical shell is based on the cylindrical coordinate system. We map the 3D cortical shell onto the $2 \mathrm{D}$ space of $(z, \varphi)$. The unwrapping process is,

$$
U(z, \varphi)=\frac{1}{\left\|S_{E}(z, \varphi)-S_{I}(z, \varphi)\right\|} \int_{S_{I}(z, \varphi)}^{S_{E}(z, \varphi)} G(z, \varphi, r) d r
$$


here $G$ is the image intensity. Essentially, we project the mean intensity of the cortical shell onto a 2D map. The mapping is one-to-one: any point on the unwrapped map has a corresponding point on the 3D cortical shell. Figure 3 a shows an example of the cortical shell unwrapped map. The horizontal axis is $\varphi$ and the vertical axis is $z$. Axis $\varphi$ starts from the center of the spinal canal (detected in section 2.1) and spans $360^{\circ}$, and axis $z$ goes from the inferior to the superior endplates.
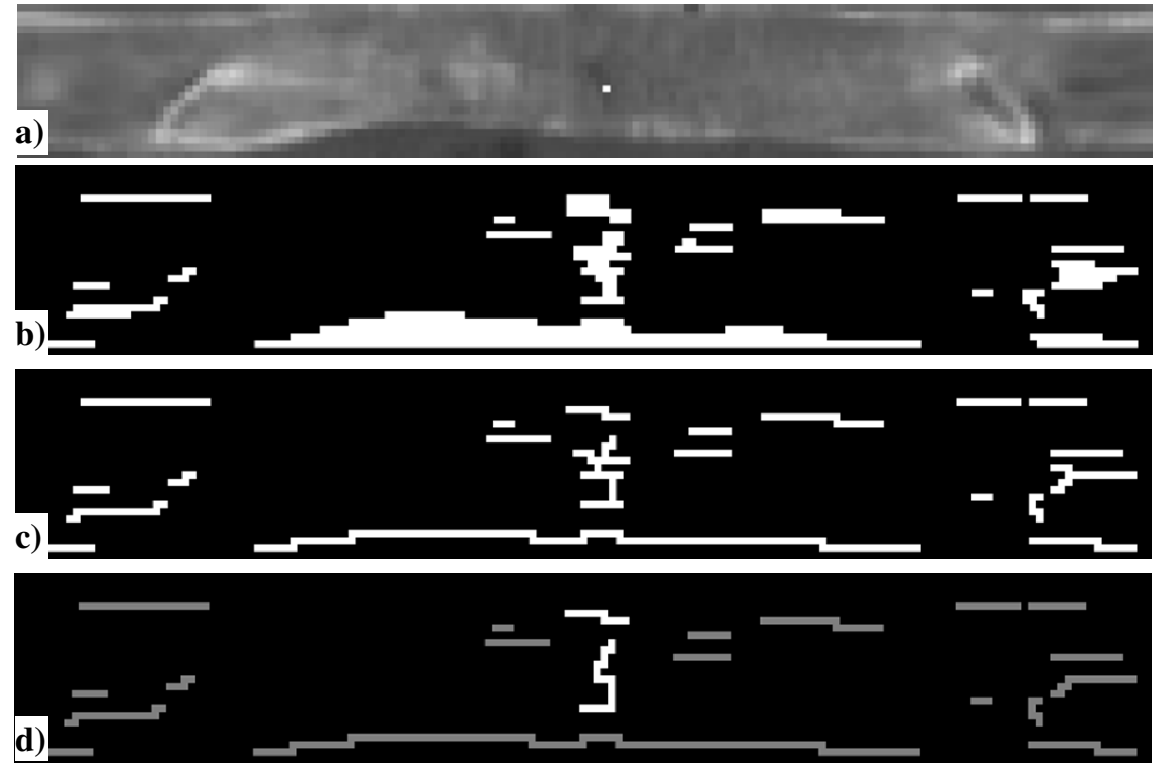

Fig. 3. Cortical shell unwrapping and fracture line detection. Original CT image is in Figure 1a. a) Unwrapped cortical shell map (bright dot indicates the projected fracture site marked by an expert on the original CT); and results after b) Adaptive filtering; c) Skeletonization; d) Pruning (the bright line is the true fracture line).

\subsection{Fracture Line Detection}

Fracture lines on the cortical shell appear as gaps or discontinuities on the unwrapped map (Figure 3a). Detecting discontinuities on the map is a relatively simple 2D pattern recognition problem compared to the complex 3D fracture detection problem, similar to road crack detection in computer vision applications [8]. We adopt a multiscale adaptive filtering method to detect cracks on the unwrapped map. Two assumptions are applied: 1) a crack is darker than the background (normal cortical shell); and 2) a crack is composed of a set of connected segments with different orientations and limited width. We define the crack filter as a rectangle function,

$$
f(x)=\left\{\begin{array}{cc}
-1 & x \in[-T / 2, T / 2] \\
1 & \text { Elsewhere }
\end{array}\right.
$$

where $T$ is the width of the crack and also the scale of the filter. We convolve the unwrapped map $U(z, \varphi)$ with $f(x)$ of different scales (by varying $T$ ) at multiple 
orientations $([0, \pi / 4, \pi / 2,3 \pi / 4])$. The outputs of all filters are merged and used as the initial detection for the fractured regions (Figure $3 b$ ). The merging is additive and a connected component analysis is conducted to obtain detections.

We then apply a Hilditch thinning algorithm [9] to skeletonize the fracture region (Fig 3c). After that, the branches on the skeleton are pruned [10] so that only the longest path remains, and is detected as one potential fracture line (Fig 3d).

\subsection{Feature Extraction and Classification}

Many false positives remain after the filtering. We extract a set of 28 quantitative features to differentiate true fracture lines from false detections. The features for each fracture line can be roughly partitioned into four categories: location (e.g. circumferential angle, distance, and orientation), shape (e.g. width, thickness, aspect ratio), intensity (e.g. intensity, contrast) and attributions of its associated vertebral body (e.g. height, radius, and intensity). Due to the page limit, here we only list the formula for a few features $\left(f_{1}\right.$ :average width, $f_{2}$ :average thickness, $f_{3}$ :average intensity, and $f_{4}$ :average interior intensity),

$$
\begin{gathered}
f_{1}=\frac{1}{\left\|\Omega_{s}\right\|} \sum_{(z, \varphi) \in \Omega_{s}} d(z, \varphi) \\
f_{2}=\frac{1}{\left\|\Omega_{A}\right\|} \sum_{(z, \varphi) \in \Omega_{A}}\left(S_{E}(z, \varphi)-S_{I}(z, \varphi)\right) \\
f_{3}=\frac{1}{\left\|\Omega_{A}\right\|} \sum_{(z, \varphi) \in \Omega_{A}} U(z, \varphi) \\
f_{4}=\frac{1}{\left\|\Omega_{A}\right\|} \sum_{(z, \varphi) \in \Omega_{A}} \int_{0}^{S_{I}(z, \varphi)} \frac{G(z, \varphi, r)}{S_{I}(z, \varphi)} d r
\end{gathered}
$$

where $\Omega_{A}$ is the set of points in the detected fracture region, $\Omega_{S}$ is the skeleton of $\Omega_{A}$, and $d$ is the distance from a skeleton point to its closest boundary point. The features are computed from both the 3D CT data and the 2D unwrapped map.

A committee of support vector machines (SVM) [11] is trained to classify the detections into true fracture lines or false ones. The training was based on reference standard of fractures marked by an expert. A forward stepwise feature selection procedure was conducted to form a seven-member committee. Each committee member had three characteristic features (features may overlap among committee members). Ten-fold cross validation was employed to evaluate the performance.

\section{$3 \quad$ Data Sets and Experimental Results}

Our cohort includes 18 trauma patients admitted to UC Irvine Medical Center between June 2009 and July 2010. The mean patient age was $51 \pm 11$ yrs (18-86yrs). There were 
13 men and 5 women. All patients were scanned on a Siemens Sensation 64 scanner. The scanning parameters were: $2 \mathrm{~mm}$ slice thickness, $120 \mathrm{kvp}$, no intravenous contrast administration, and convolution kernel B40f (16 patients) or B60f (2 patients). The CT data covered the thoracic and lumbar spines, and included 14 vertebrae on average. An expert radiologist examined the cases and manually marked the fracture sites. Ten patients were positive for vertebral body fractures. The total number of spatially distinct fracture sites was 21 , among the 10 patients. The remaining 8 patients had no evidence of vertebral body fracture. The average running time is 5.6 minutes.

a)

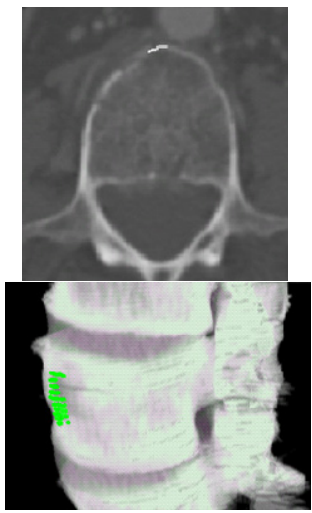

b)

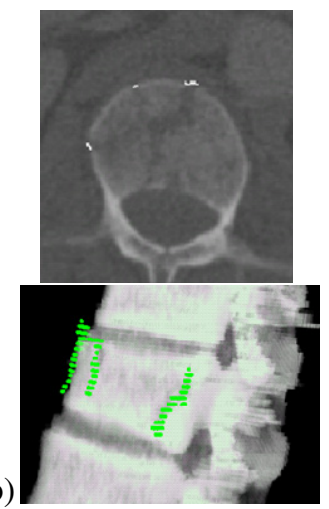

c)

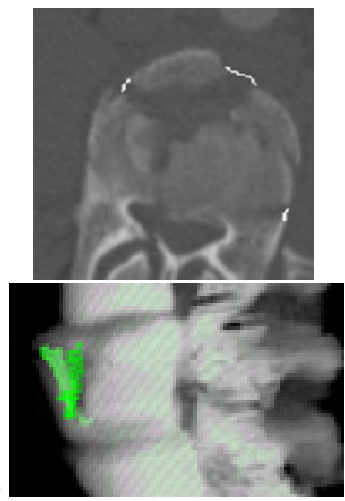

Fig. 4. Examples of detected fracture lines. Original images are in Figure 1. First row: 2D view; second row: $3 \mathrm{D}$ view.

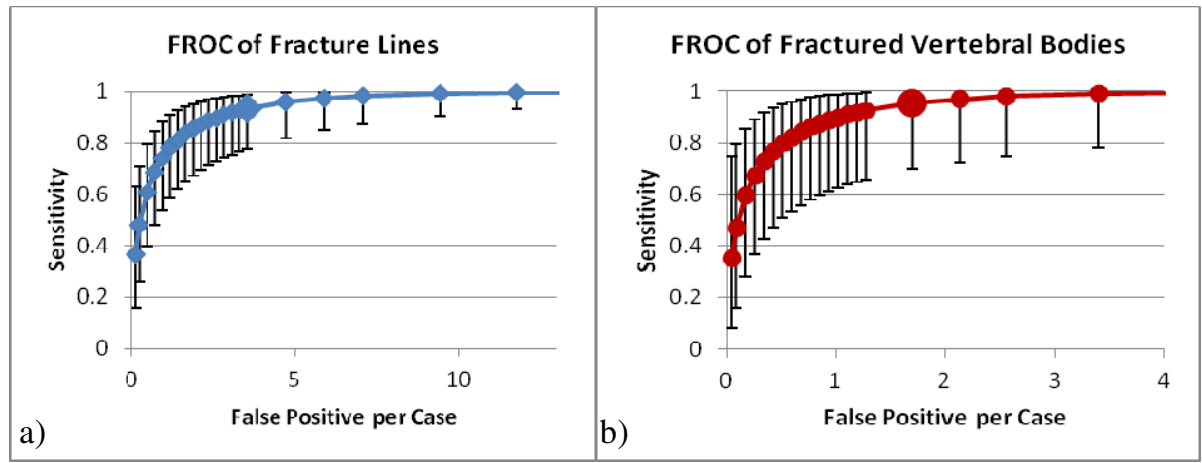

Fig. 5. FROC analysis. Big markers indicate operating points.

Figure 4 shows examples of detected fracture lines. Our method successfully detected both nondisplaced and simple fractures (Fig. 4a, 4b), as well as burst fractures (Fig. 4c). Figure 5a shows the FROC curve of fracture line detection. We also evaluated the performance on a per-vertebra basis, by merging all detections in one vertebral body into one "detection" (only keeping the detection with highest SVM value) and report the number of vertebrae having any fracture (FROC in Figure 5b). The system achieved $92.7 \%$ sensitivity (95\% confidence interval: $[76.7 \%, 98.5 \%]$ ) at 3.3 false fracture sites per patient, and $95.3 \%$ sensitivity $(95 \% \mathrm{CI}:[72.6 \%, 99.9 \%])$ at 
1.7 false fractured vertebrae per patient. The FROC analysis was conducted using ROCKIT (http://xray.bsd.uchicago.edu/krl/KRL_ROC/software_index6.htm).

The etiology of common false positives includes costovertebral junctions, partial volume averaging of vertebral disks and nutrient vessel foramen.

\section{Discussion}

Our method converts a complex 3D fracture detection problem into a simpler $2 \mathrm{D}$ pattern recognition problem and achieves high sensitivity and specificity. The system may serve as a shadow reader to assist radiologists and has the potential to increase detection rates for spinal fractures at early points after occurrence, allowing appropriate management and preventing secondary complications.

Future work will include detection of fractures in spinous and transverse processes. We will also incorporate rib segmentation in the system to reduce the number of false positives at the costovertebral junctions.

\section{References}

1. Smith, M., et al.: The Reliability of Nonreconstructed Computerized Tomographic Scans of the Abdomen and Pelvis in Detecting Thoracolumbar Spine Injuries in Blunt Trauma. J. Bone Joint Surg. Am. 91, 2342-2349 (2009)

2. He, J.C., Leow, W.-K., Howe, T.S.: Hierarchical Classifiers for Detection of Fractures in X-Ray Images. In: Kropatsch, W.G., Kampel, M., Hanbury, A. (eds.) CAIP 2007. LNCS, vol. 4673, pp. 962-969. Springer, Heidelberg (2007)

3. Guglielmi, G., et al.: Assessment of osteoporotic vertebral fractures using specialized workflow software for 6-point morphometry. European Journal of Radiology 70(1), 142-148 (2009)

4. Ghosh, S., et al.: Automatic Lumbar Vertebra Segmentation from clinical CT for Wedge Compression Fracture Diagnosis. In: SPIE Medical Imaging (2011)

5. Yao, J., et al.: Quantitative Vertebral Compression Fracture Evaluation Using a Height Compass. In: SPIE Medical Imaging (2012)

6. Yao, J., O’Connor, S.D., Summers, R.M.: Automated Spinal Column Extraction and Partitioning. In: IEEE ISBI, Arlington, VA (2006)

7. Xu, C., Pham, D.L., Prince, J.L.: Medical Image Segmentation Using Deformable Models. In: Sonka, M., Fitzpatrick, J.M. (eds.) Handbook of Medical Imaging, Medical Image Processing and Analysis, vol. 2, pp. 129-174. SPIE (2000)

8. Chambon, S., et al.: Road crack extraction with adapted filtering and Markov model-based segmentation. In: International Joint Conference on Computer Vision Theory and Applications, Angers, France (2010)

9. Zhang, T.Y., Suen, C.Y.: A Fast Parallel Algorithms For Thinning Digital Patterns. Communication of the ACM 27(3), 236-239 (1984)

10. Bai, X., Latecki, L.J., Liu, W.-Y.: Skeleton Pruning by Contour Partitioning with Discrete Curve Evolution. IEEE Transactions on Pattern Analysis and Machine Intelligence 29(3), 1-14 (2007)

11. Cristianini, N., Taylor, J.S.: An Introduction to Support Vector Machines. Cambridge University Press (2000) 